Review

\title{
A historical perspective on sacral nerve stimulation (SNS) for bowel dysfunction.
}

\author{
Elroy Patrick Weledji
}

Department of Surgery, Faculty of Health sciences, University of Buea, Cameroon

Correspondence: E.P. Weledji, Pemset House, Lumpsum quarters, Limbe, S.W. Region, Cameroon. E-mail: elroypat@yahoo.co.uk; Tel 237699922144

Running title: Sacral nerve stimulation for bowel dysfunction

\begin{abstract}
Prevention of obstetric trauma from damage to the pelvic floor is not always possible and sacral nerve stimulation (SNS) may be necessary later in life. Sacral nerve stimulation has been a promising innovation in the management of moderate to severe faecal incontinence and following sphincter repair failure. Although the indication spectrum for SNS is expanding, the success of neuromodulation for constipation is limited. Adverse events of SNS requiring reintervention are not common but a long-term successful outcome may depend on interventions for maintenance of the device.
\end{abstract}

Keywords: pelvic floor, bowel, dysfunction, sacral nerve, stimulation

\section{Background}

The commonest cause of faecal incontinence in women is obstetric trauma (table 1) [1,2]. Urge incontinence is a marker of severe external anal sphincter dysfunction associated with reduction in voluntary squeeze pressure and multiple vaginal deliveries $[1,2]$. The original idea of nerve stimulation came from the improved anal squeeze pressure following the stimulation of a portion of the pudendal nerve (S2-4) below the ischial spine. The sacral nerve roots (S3 or S4) were however the easiest to access (figure 1) and, the first stimulators for sacral nerve stimulation (SNS) for urinary urge incontinence and non-obstructive urinary retention were implanted by Tanagho and Schmidt in 1988 [3]. Observations have noted benefits beyond voiding disorders which include re-establishment of pelvic floor muscle awareness, resolution of pelvic floor muscle tension and pain, and normalization of bowel function [4]. Trials on patients with functional deficits but an intact external anal sphincter and, faecal incontinence (FI) resistant to conservative treatments (anti-diarrhoeals, biofeedback) were extremely encouraging [4, 5]. The indication spectrum evolved in the following years and patients with limited sphincteric damage, idiopathic sphincter degeneration, partial spinal cord injury, irritable bowel syndrome (IBS), scleroderma, rectopexy or low anterior resection benefited from the use of SNS [4]. Thus, the aetiology of FI and the extent of sphincter damage did not seem to limit treatment success [6-9]. Although more recently some benefit is being shown in patients with both slow and normal transit constipation, a Cochrane review by Thaha et al concluded that SNS did not improve symptoms in patients with constipation [10]. Long-term outcome may be predicted from clinical 
symptomatology and manometric neurophysiological testing before surgery in idiopathic faecal incontinence although there is no clear correlation. Anorectal investigation has revealed a large group of parous women with occult sphincter trauma which may have a clinical impact as these women get older [2]. SNS had become a recognized treatment option for faecal incontinence with more than 800 implants in Europe at 2003 and 340,000 implants in both urology and coloproctology in 2021 [11-14]. The establishment of national registries was important for quality assurance and the further development of this therapy [11].

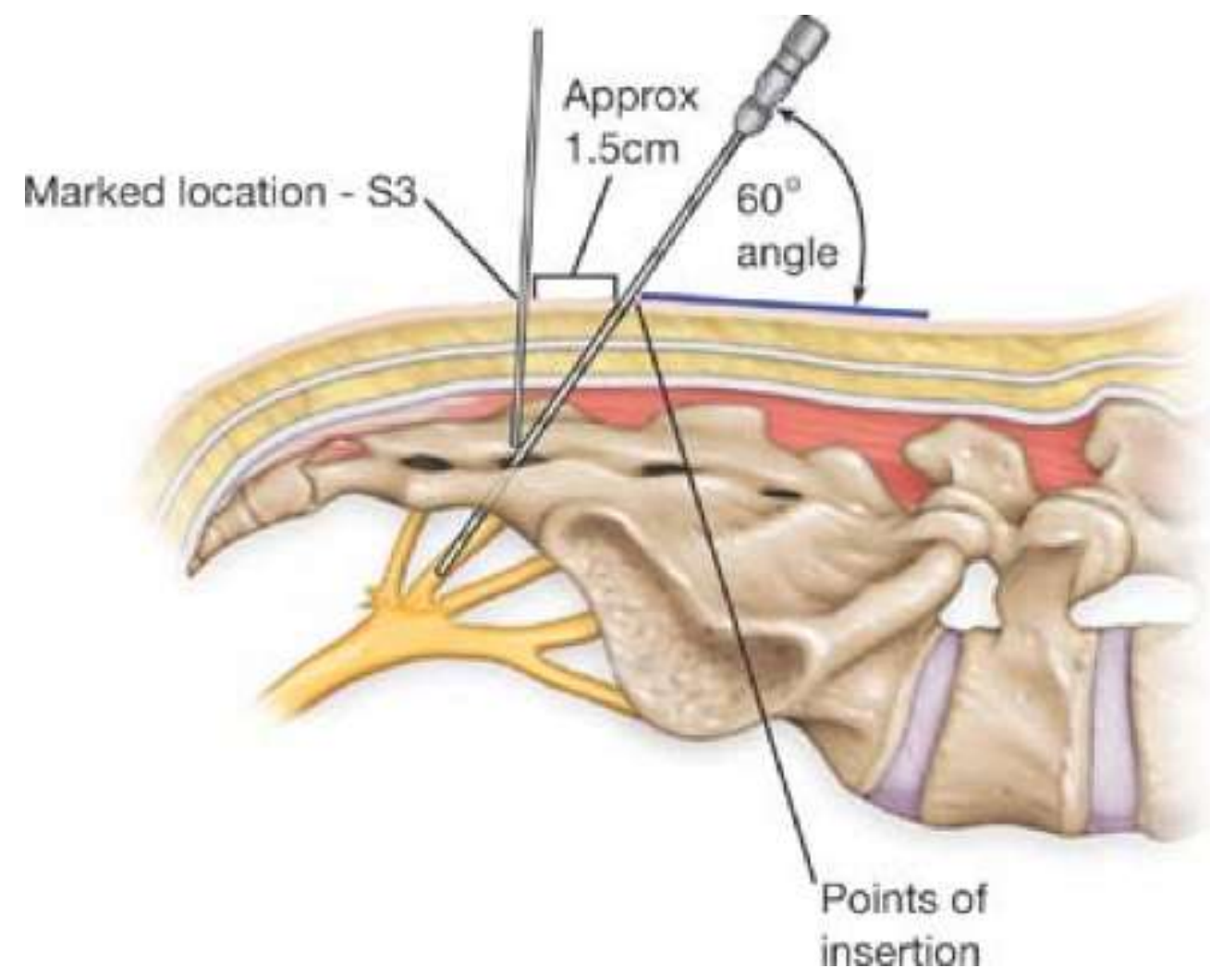

Figure 1. Schematic diagram of sacral nerve stimulation

Table 1. Aetiology of faecal incontinence [4]

\begin{tabular}{ll}
\hline Trauma & Obstetric, Surgical, Accidental/war injury, \\
\hline Colorectal disease & $\begin{array}{l}\text { Haemorrhoids, Rectal prolapse, Inflammatory } \\
\text { bowel disease }\end{array}$ \\
Congenital & $\begin{array}{l}\text { Spinal bifida, Operations for imperforate anus, } \\
\text { Hirschsprung's disease }\end{array}$ \\
Neurological & Cerebral, Spinal, Peripheral \\
Miscellaneous & Behavioural, Impaction, Encopresis \\
\hline
\end{tabular}

\section{Anatomical and Physiological background of Sacral Nerve Stimulation}

The hindgut and continence mechanisms are influenced by both intrinsic and extrinsic nerve supplies. Somatic (extrinsic) nerve supply to the continence mechanism acts on both the external 
anal sphincter and levator ani muscles (S 2-4) which during acute sacral nerve stimulation testing contract causing a 'bellows' movement in the perineum (contraction and lifting of the anus and pelvic floor) . Intrinsic nerve supply in the bowel wall is found in the submucosal (Meissner's) and the myenteric (Auerbach's) plexuses that controls the contractile and some secretory functions of the bowel. Extrinsic autonomic nerves (sympathetic and parasympathetic) are modulatory and act upon the intrinsic system. Sympathetic innervations arises from T1-L2 and via the sympathetic chain and inferior mesenteric and hypogastric plexuses reinforced by lumbar and sacral sympathetic splanchnics inhibits colonic motility and stimulates the internal anal sphincter. Parasympathetic supply arises from S 2-4 to form the pelvic splanchnic nerves and via the superior and inferior hypogastric plexuses has the opposite effect on the hindgut and internal anal sphincter respectively. These two parts of the autonomic system act in a balanced and reciprocal fashion with activity being the result of the two opposing forces. The anatomical and physiological convergence of the somatic and autonomic nerves occurs at the sacral level. The sacral nerves are a mixed nerve with the largest fibres with the lowest threshold being the 1a sensory fibres followed by the alpha- motoneurones. Hence, on acute testing external sphincter contraction and a 'bellows' movement is seen. In long term stimulation an increase in maximal squeeze pressure or duration of voluntary contraction may be expected. Stimulation of the autonomic nervous system might be expected to alter internal anal sphincter function and hence resting pressure as well as affecting hind-gut motility. Although no consistent rise in resting pressure is seen 24 hour ambulatory manometry studies did show change in hindgut and anal function. Afferent sensory pathways are poorly defined and accompany autonomic fibres. On physiological testing a significant improvement in rectal sensation is observed following SNS. The intermediate transition zone of the anal canal is just above the dentate line and plays a central role in sensory function and eliciting continence. The rectoanal inhibitory reflex in which increasing rectal distension is associated with transient reflex relaxation of the internal anal sphincter and contraction of the external anal sphincter enables the discrimination between solid, liquid and flatus. Although the mechanism of action of SNS remains elusive, it is likely that the modulation of the somatic fibres to the external anal sphincter and pelvic floor, autonomic fibres to the internal anal sphincter and colon and the afferent sensory fibres from the anus and rectum confers the benefit to patients. The relative contributions and the spinal and supraspinal effect of stimulation on the higher centres remain to be elucidated $[13,15]$.

\section{Management of faecal incontinence}

The management of faecal incontinence would depend on the aetiology. Most patients who have a specific sphincter defect following obstetric or direct injury identified by three-dimensional manometry or endoanal ultrasound are best served by surgical repair. However, surgical options are limited as many patients do not have a sphincter defect amenable to repair [13]. In addition, sphincter repair procedures, post anal repair and total pelvic floor repair have proved ineffective in the long-term $[4,13]$. Those with severe neuropathic incontinence are best treated by intensive conservative management with control of stool consistency and physiotherapy or pelvic floor retraining (biofeedback) before surgical treatment is considered [16]. Biofeedback (behavioural) treatment is particularly useful in patients who have primarily a sensory problem in the anal canal leading to insensible loss of faeces. Patients are trained using either electromyographic or manometric feedback to improve the strength of their anal sphincters and if 
coupled with an intrarectal balloon, may improve their rectal sensory awareness [16]. More specific physiotherapy using either interferential treatment of the pelvic floor and, in particular trophic stimulation via anal plug electrode which uses electrical impulses designed to mimic the train of signals along the pudendal nerve can be extremely successful. The precise role of electrical stimulation of the pelvic floor is unclear, but the results are almost equivalent to those obtained by post anal repair [17]. Biofeedback training cause improvement of $70 \%$ of patients and can improve patients with structural defects of the anal sphincters [4, 17]. A proportion with persistent severe incontinence required more intensive treatment and direct surgical intervention had been the next step. External anal sphincter defects may be dealt with by anterior overlapping sphincter repair. Early results showed good symptomatic improvement in 70-80\% of patients [4]. However, long term follow up showed that results deteriorated with time. This is because, although the primary problem may have been a specific sphincter defect, an additional levatorplasty was necessary as many of these patients will have a degree of coexisting neuropathic damage to the pelvic floor, particularly following multiple vaginal deliveries $\cdot 2,19]$. Although the presence of neuropathic damage in these patients did not alter the initial surgical management it did influence prognosis as seen in the poor long-term benefit from post anal repair or a total pelvic floor repair [18]. Parks in 1975 [20] had developed the post anal repair to re-establish the normal $90^{\circ}$ anorectal angle which is a key factor in the continence mechanism. This was obtuse in many patients with idiopathic faecal incontinence. However, pudendal nerve terminal motor latency was the only preoperative variable that correlated with long-term outcome of post anal repair confirming the role of pudendal nerve damage. [11,13]. Although the early results were encouraging, the progression of pudendal nerve damage over time led to disappointing long-term results following post anal repair for neurogenic incontinence [17, 19]. The aetiology of this progressive denervation is not known. The operation of total pelvic floor repair was devised in response to the fact that many patients had associated anatomical defects such as rectocele and abnormal perineal descent. Results from total pelvic floor repair were initially encouraging, with $90 \%$ of patients either being fully continent or with improved continence. However, long term follow-up of a group of 57 patients revealed that only $14 \%$ were rendered completely continent and social activity remained compromised in $76 \%$ [17]. Since the advent of sacral nerve stimulation (SNS) total pelvic floor repair has become obsolete. The indications for stimulated neosphincter formation are expanding but dynamic graciloplasty and artificial bowel sphincter implants require major surgery and have a high morbidity and failure rate [4].The magnetic sphincter augmentation device for adult faecal incontinence has much greater morbidity than SNS [13]. The placement of a permanent stoma has also always been an option $[1,4]$.

\section{Sacral Nerve Stimulation}

SNS is an expensive therapy that requires a dedicated team for an optimal outcome [4]. When SNS was first used for patients in the field of urology it was noted that patients with concomitant faecal incontinence derived benefit. Following further studies, a similar technique utilized for faecal incontinence showed promising results [5-12, 21-38]. In addition, the technique of test stimulation prior to full implantation offered a unique advantage over other treatment options. A trial of SNS was justified for patients in whom the primary aetiology appeared to be neurogenic and in whom preoperative investigations had failed to reveal a sphincter defect. To qualify for 
assessment for SNS, the patient should have at least one episode of faecal incontinence per week to either solid or liquid stool. They should have failed maximal medical therapy, including the use of anti-diarrhoeal medication and a course of biofeedback treatment [4, 13]. Specific inclusion and exclusion criteria are outlined in table $2[4,21]$. Prior to stimulation all patients would complete a bowel habit diary for a two or three week period. As treatment intends to improve quality of life, a health survey quality of life questionnaire for faecal incontinence [21] are helpful instruments to assess the impact of SNS on quality of life. Endoanal ultrasound and anorectal physiological testing are performed in each patient to assess any structural or functional problem of the continence mechanism. The neuromuscular axis needed to be intact as shown by a residual voluntary function and/or reflex action of the external anal sphincter. The key advantages of the neuromodulation technique include (1) a conscious sedation which allows the patient to provide sensory feedback, (2) the control of the lead position with the radiopaque markers by means of fluoroscopy, (3) the small surgical incision and minimal muscle trauma, and (4) a reduced surgical time. On deciding to proceed with SNS, there is the benefit of being able to undertake a test stimulation period with either a temporary or permanent electrode implant attached to an external pulse generator. The test stimulation period thus allows optimization of patient selection prior to implantation of the definitive implantable nerve stimulator (INS). The newly developed 'tined lead' allows minimally invasive placement reducing operation time, in patient stay and patient discomfort. A percutaneous wire is inserted under general or local anaesthesia into the second, third or fourth sacral foramen [13, 35-37]. The S3 nerve contains efferent and afferent fibres from the anterior part of the levator ani and sensory fibres from the perineum and the genitalia. There is the distinct sensation of perforating the rigid ligamentous structures as compared with hitting the periosteum of the sacrum when entering the dorsal opening of the foramen. The angle of insertion should be acute $\left(60^{\circ}\right.$ at the level of the skin) to minimize the risk of nerve or vascular damage and as the needle has to cross soft tissue before entering the foramen, its entrance point should be cephalad to the position of the foramen (figure 1). Gentle movements in millimeter steps with intermittent stimulation of graduated amplitude will help optimize positioning. Markers on the needle electrode indicate the depth of placement. A motor response of the pelvic floor and the anus (if general anaesthesia) or a sensory response (if local anaesthesia) will optimize the positioning of the needle. S2 stimulation results in a clamp-like contraction of the perineal muscles and an outward rotation of the ipsilateral leg. S3 stimulation leads to contraction of the levator ani and external anal sphincter, resulting in a bellows-like movement and circular contraction of the anus, along with plantar flexion of the first and second toes. S4 affects the posterior part of levator ani with sensation around the anus and has no motor effect on the legs $[35,36]$. It thus produces a bellows-like contraction of the levator ani and circular contraction of the anus without movement of leg, foot, or toe. There is no recommendation on S3 or S4 stimulation [37] as a study showed no difference in the effect on incontinence [38]. A successful position is manifested by contraction of the pelvic floor and flexion of the ipsilateral great toe. The wire is connected to an external stimulator and the results monitored for a period of 2-3 weeks. If successful, a permanent indwelling stimulator can be inserted. Patient selection for permanent implantation is based on the degree of improvement in continence as ascertained from bowel diaries completed before and during temporary stimulation and patient satisfaction. A 50\% or greater improvement in either the total number of faecal incontinent episodes or in the number of days affected by incontinent episodes are the recommended success criteria in order to proceed to permanent implantation. [9, 23]. A permanent tined electrode is implanted via a percutaneous approach. 
The implantable nerve stimulator (INS) is connected and inserted into a subcutaneous pocket created in the ipsilateral buttock and the settings can be altered transcutaneously by the clinician or patient (figure 2). The programmer communicates non-invasively by means of a radiofrequency signal with the INS which can be easily used in the operating room and the clinic due to its compact size. The technical development will continue to further minimize the invasiveness and increase the effectiveness. This will include smaller sized pulse generators with a direct connect lead and enhancements to the physician as well as the patient programmer. Physicians will eventually be able to download several programs onto the patient programmer to allow the patient to toggle between different programmes to achieve optimal therapy benefit. Linking diagnostic procedures with SNS may also play an important role in the further development of this therapy. SNS has developed over the past decades not only in terms of indications, but also with regard to the technique itself. These technical innovations resulted in a less invasive procedure for patients and an easier and more reliable approach for physicians. The test stimulation is associated with a very low level of complications, and a complication rate of $5-10 \%$ for insertion of a permanent implant [7]. Initial reports suggested improvement of continence in approximately $70-80 \%$ of patients who permanently implanted [4-7, 9, 27]. Bilateral stimulation may provide a solution for patients who have not benefited from or have not had optimal results with unilateral stimulation [34]. As the indication spectrum is evolving the invasiveness of the procedure is being reduced. Most re-interventions are due to expected maintenance rather than to adverse events requiring operative revisions. All patients with nonrechargeable INSs need a battery change after a number of years. However, re-operations for complications are worthwhile as they sustain the treatment success [40].

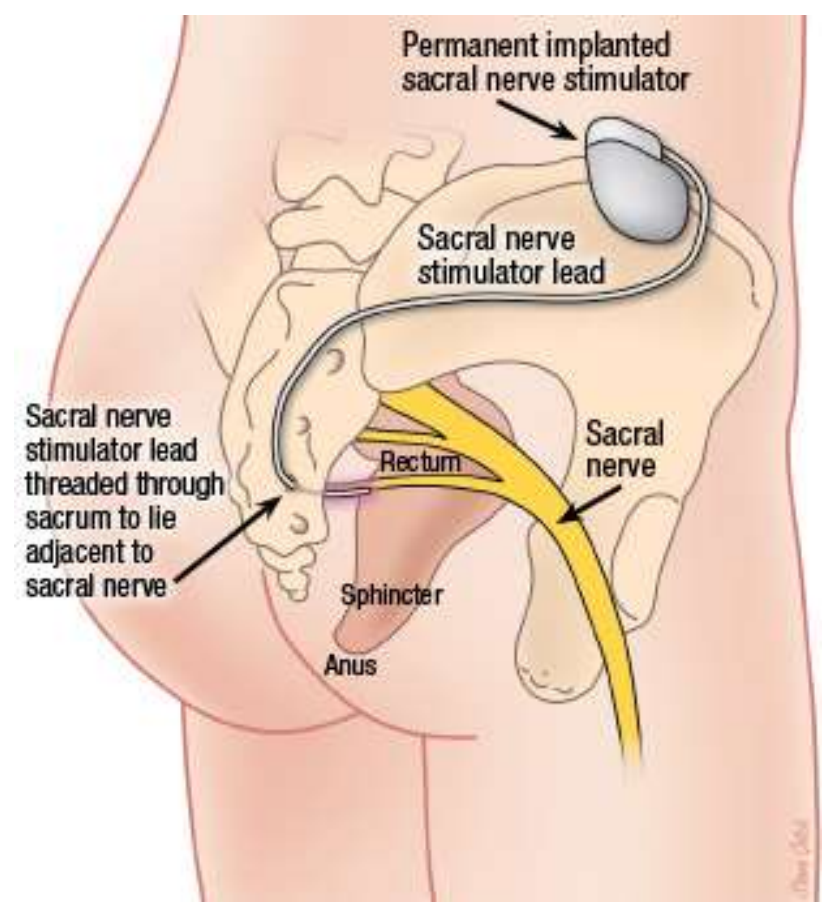

Figure 2: Schematic diagram of sacral nerve stimulation with permanent implanted sacral nerve stimulator 
Table 2. Patient selection criteria [4]

\begin{tabular}{ll}
\hline Indication & $\begin{array}{l}\text { Urge \& Passive incontinence, } \\
\text { Intact external sphincter }+/ \text { - previous repair, } \\
\text { failed conservative management }\end{array}$ \\
\hline Absolute exclusion criteria & Skin disease (Pilonidal sepsis), Pregnancy \\
Probable good results & Congenital malformation, rectal resection \\
With care & EAS defects (especially longstanding) \\
\hline
\end{tabular}

\section{Critical appraisal of earlier published data}

Clinical evaluation of SNS was initiated with Matzel et al [11] in Germany publishing the first case series in 1995. At 6 months follow-up of SNS in 3 patients with FI, full continence was achieved in 2 patients and one patient improved from daily urge incontinence to passive soiling only. Later published studies had reported excellent clinical results and very few complications [28-33]. These included pain at the electrode or pulse generator site [29], infection and recurrent lead dislodgement [31], superficial wound dehiscence and lead migration [33], and these complications required explantation. Changes in manometric data have not been consistent between series and on a case-by-case basis but a significant increase in squeeze and resting pressure was noted in a number of publications [31-35]. There was a significant increase in rectal volume tolerability and rectal sensitivity following successful treatment with SNS for faecal incontinence. A significant increase in anal resting pressure controlled by the autonomic nerves, but not in maximum squeeze pressure suggests that SNS causes neuromodulation at the spinal level [36]. Standardized methods of evaluation were applied to refine accurate patient characterization for SNS. The technique appeared to benefit patients who had an intact anal sphincter on ultrasound and some preservation of pudendal nerve function. Earlier studies had demonstrated the benefit of sacral neuromodulation in patients with spinal cord injuries [31]. This corroborates the fact that a morphologically intact anal sphincter is not necessary for success with sacral nerve stimulation in patients with FI [32]. The short and medium-term success of SNS in patients with FI have been demonstrated in several studies [37-39]. Earlier studies demonstrated that in the medium term sacral nerve stimulation is an effective option of patients with neuropathic incontinence who had failed conservative treatment and whose only viable alternative option would be a stoma [24-27]. Although SNS was accepted by the U.S. Food and Drug administration (FDA) in 2011, longer- term studies in the United States (USA) in larger number of patients with reasonable follow-up ( $>$ 3years) confirmed an efficacy of about 50\%. [34]. For young patients who have severe sphincter injuries, a failed sphincter repair, or insufficient residual sphincter consideration is given to either SNS or some form of sphincter augmentation or artificial bowel sphincter [4]. The current evidence suggests a high efficacy of SNS along with a low complication rate in FI regardless of the aetiology [6, 24, 4148]. There is a success rate of $66.8 \%-90 \%$ [44, 47, 48] over a follow-up period of over 3 years $[43,44,47]$. A reduction of $>50 \%$ in involuntary evacuations per week is described in $75-84 \%$ of patients [37, 46] with a Wexner score reduction from 16 to 7 [43]. Although long term follow-up with a large number of patients are rare studies with long-term follow-up have shown lasting effect [47, 48]. Widmann et al [39] in 2019 demonstrated 70\% successful outcome in 79 patients seven years after implantation. Although re-interventions due to complications were necessary for 24 patients $(30 \%)$, these patients had a 5 -year success rate of $89 \%$ compared to 
$78 \%$ for patients without intervention. A lead position at S3 was associated with an improved outcome. However, the largest recent multicentre study by Altomare et al [45] that included 222 patients showed a 5 -year success rate of 54\%. The better outcome in the Widmann et al's study may be due to the rigorous follow-up with the consequent maintenance intervention [39]. Adverse events requiring operative revision were not common but mechanical lead damage, dislodgement or migration required re-intervention. The fact that pelvic floor functioning rely on a complex interplay between various mechanisms may be a challenge to the procedure with regard to patient selection and efficacy. Nevertheless, SNS is gaining an increasing role in treatment of bowel disorders for whom therapy was limited $[4,46.49 .50]$ including bowel dysfunction from anorectal malformation [51,52]. SNS is effective in patients with FI after surgery for anal atresia [52] as well as those with LARS (low anterior resection syndrome) [46]. Ramage et al [46] concluded in their systematic review that sacral nerve stimulation for LARS was effective in $75 \%$ and $94 \%$ at intention-to-treat analysis and per-protocol analysis, respectively. Constipation is an area in which SNS's role has been investigated and earlier reports of a pan-European study had been encouraging with approximately $60 \%$ of patients selected for temporary implant going onto successful permanent implantation [37]. However, when a multicentre prospective consecutive cohort study evaluated the efficacy of SNS in patients with idiopathic constipation, only those with slow or normal transit but impaired evacuation benefited [53]. Current studies described $\sim 30 \%$ response of SNS for refractory constipation, although a small group of patients were highly satisfied [54]. Although mediumterm success rates of up to $90 \%$ have been reported, [55] the overall results are disappointing especially as the efficacy deteriorates with time [37, 39]. The outcome seems better when FI is accompanied by constipation [39, 56]. As SNS therapy for constipation is less effective than for FI [39, 57], further trials are suggested [58]. Early results from SNS for severe irritable bowel syndrome (IBS) are promising which indicates evidence of pelvic floor dyssynergia in IBS [5860]. Although SNS is a relatively expensive procedure, a study indicated SNS treatment for IBS being cost-effective from a 7 -year perspective onwards [61].

\section{Conclusions}

SNS is a minimally invasive procedure that offers a real alternative to patients with refractory pelvic floor dysfunctions. Its safety profile is very favourable. Although the mechanism of action remains unknown, it offers an effective sustainability treatment for FI especially the majority of patients with neuropathic faecal incontinence. Re-interventions are worthwhile as they sustain the treatment success. The success of SNS for constipation is, however, limited.

\section{Conflict of Interest}

The authors declare that there is no conflict of interest.

\section{References}

1. Wald, A. "Fecal incontinence in adults". N Engl J Med 2007; 356:1648-1655 
2. Guise, J.M, Morris, C, Osterweil, P; et al." Incidence of fecal incontinence after child birth". Obstet Gyn 2007; 109:281-8

3. Tanagho, E.A. Schmidt, R.A. "Electrical stimulation in the clinical management of the neurogenic bladder". The J of Urology 1988; 14096:1331-1339

4. National institute of Clinical Excellence (NICE) 2013. "Faecal incontinence: the management of faecal incontinence in adults. Clinical guidelines $C G$ ": 49 , National Institute for Health and care Excellence (NICE).

5. Rosen, H.R., Urbarz, C, Holzer, B., et al."Sacral nerve stimulation as a treatment for fecal incontinence". Gastroenterology 2001; 121:536-41

6. Boyle DJ, Knowles CH, Lunniss PJ, et al. "Efficacy of sacral nerve stimulation for fecal incontinence in patients with anal sphincter defects ". Dis Colon Rectum 2009 52:1234-1239.

7. Altomare, D.F.. Giuratrabocchetta, S., Knowles, C.H., et al. " Long-term outcomes of sacral nerve stimulation for faecal incontinence”. Br J Surg 2015;102:407-415

8. Gourcerol G, Gallas S, Michot F, et al. "Sacral nerve stimulation in fecal incontinence: are there factors associated with success"? Dis Colon Rectum 2007; 50:3-12.

9. Roy AL, Gourcerol G, Menard JF, et al. 'Predictive factors for successful sacral nerve stimulation in the treatment of fecal incontinence: lessons from a comprehensive treatment assessment". Dis Colon Rectum 2014; 57:772-780

10. Thaha MA, Abukar AA, Thin NN, et al. Sacral nerve stimulation for faecal incontinence and constipation in adults. Cochrane Database Syst Rev 2015: 8: CD 004464)

11. Matzel KE, Stadelmaier U, Hohenfellner M, et al. Electrical stimulation of sacral spinal nerves for treatment of faecal incontinence. Lancet 1995;346: 1124-1127

12. Ganio E, Lue AR, Clerico G et al. Sacral nerve stimulation for treatment of faecal incontinence. A novel approach for intractable fecal incontinence. Dis Colon Rectum 2001;44:619-31

13. Jayne PG, Williams AE, Corrigen N, Croft J, Pillan A, Napp V, et al. Sacral nerve stimulation versus the magnetic sphincter augmentation device for adult faecal incontinence. Health Technol Assess 2021;25(18):1-96

14. Hernandez-Hernandez, D, Padilla- Fernandez B, Romera CM, Medler HS, CastroDiaz, D. Long-term outcomes of sacral nerve stimulation in pelvic floor dysfunctions. Int Neurourol J 2021: ahead of print doi;10.5213/inj.2040364.182

15. Weledji EP. The electrophysiological basis of faecal incontinence and its implications for treatment. Annals of Coloproctology 2017;33:161-168

16. Cooper EA, Df- Loyde KJ, Young CJ, et al. Pudendal nerve testing does not contribute to surgical decision making following anorectal testing in patients with faecal incontinence. Int J. Colorectal Dis 2016;31:1437-42

17. Norton C, Chelvanayagam S, Wilson-Barnet J et al. Randomised control trial of biofeedback for fecal incontinence. Gastroenterology 2003; 125: 1320-9

18. Pinho M, Ortiz J, Oya M et al. Total pelvic floor repair for the treatment of neuropathic fecal incontinence. Am J Surg 1992;163:340-3

19. Weledji EP, Elong A, Verla V. Secondary repair of severe chronic fourth-degree perineal tear due to obstetric trauma. J of Surg Case Rep 2014;2

20. Parks AG. Anorectal incontinence. Proc R Soc Med 1975; 68:681-90 
21. Roy AL, Gourcerol G, Menaed JF, et al. Predictive factors for successful sacral nerve stimulation in the treatment of fecal incontinence: lessons from a comprehensive treatment assessment. Dis Colon Rectum 2014;5: 772-80

22. Rockwood TH, Church JM, Fleshman JW, et al. Fecal Incontinence Quality of life Scale: quality of life instrument for patients with fecal incontinence. Dis Colon Rectum 2000;43:9-16

23. Kenefick NJ, Christiansen J. A review of sacral nerve stimulation for the treatment of faecal incontinence. Colorectal Dis 2004; 6:75-80

24. Kenefick NJ, Vaizey CJ, Cohen R, et al. Medium-term results of permanent sacral nerve stimulation for faecal incontinence. Br J Surg 2002;89:896-901

25. Melenhorst J, Koch SM, Uludag O et al. Sacral neuromodulation in patients with faecal incontinence: results of the first 100 permanent implantations. Colorectal Dis 2007;9:725-30.

26. Malouf AZ, Vaizey CJ, Nicholls RJ et al. Permanent sacral nerve stimulation for fecal incontinence. Ann Surg 2000; 232:143-8

27. Rosen HR, Urbarz C, Holzer B, et al. Sacral nerve stimulation as a treatment for fecal incontinence. Gastroenterology 2002;121:536-41

28. Ganio E, Ratto C, Masin A, et al. Neuromodulation for fecal incontinence: Outcome in 16 patients with definitive implant. Dis Colon Rectum 2001;44:965-970

29. Leroi AM, Michot F, Grise P, et al. Effect of sacral nerve stimulation in patients with fecal and urinary incontinence. Dis Colon Rectum 2001;44:779-789

30. Uludag O, Darby M, Dejong C, et al. Sacral neuromodulation: effective in the treatment of faecal incontinence with intact sphincter muscles; a prospective study. Ned Tijdschr Geneeskd. 2002;146:989-93

31. Jarrett ME, Matzel KF, Christiansen J et al. Sacral nerve stimulation of faecal incontinence in patients with previous partial spinal injury including disc prolapse. $\mathrm{Br}$ J Surg 2005;92:734-9

32. Melenhorst J, Koch SM, Uludag O, et al. Is a morphologically intact anal sphincter necessary for success with sacral nerve modulation in patients with faecal incontinence? Colorectal Dis.2008;10:257-262

33. Matzel KE, Stadelmaier U, Bittorf B, et al. Bilateral sacral spinal nerve stimulation for fecal incontinence after low anterior rectum resection. Int J Colorectal dis 2002; $17: 430-434$

34. Hull T, Giese C, Wexner SD, et al. SNS Study group. Long-term durability of sacral nerve stimulation therapy for chronic fecal incontinence. Dis Colon Rectum 2013;56:234-45

35. Dijkema HE, Weil EH, Mijs PT, et al. Neuromodulation of sacral nerves for incontinence and voiding dysfunctions. Clinical results and complications. Eur Urol 1993;24:72-76.

36. Schmidt RA, Senn E, Tanagho EA. Functional evaluation of sacral nerve root integrity. Report of a technique. Urology 1990;35:388-392.

37. Maeda Y, O’Connell PR, Lehur PA, et al. European SNS Bowel Study Group. Sacral nerve stimulation for faecal incontinence and constipation: a European consensus statement. Colorectal Dis 2015;17:O74-O87.

38. Patton V, Abraham E, Lubowski DZ. Sacral nerve stimulation for faecal incontinence: medium-term follow-up from a single institution. ANZ J Surg 2017;86:462-466. 
39. Widmann B, Galata C, Warschkow R, et al. Success and complication rates after sacral neuromodulation for feacal incontinence and constipation: a single centre follow-up study. J Neurogastroenterol Motil 2019;25:1

40. Michelsen HB, Buntzen S, Krogh K, et al. Rectal volume tolerability and anal pressures in patients with fecal incontinence treated with sacral nerve stimulation. Dis Colon Rectum 2006;49: 1039-44

41. Hotouras A, Murphy J, Thin NN, et al. Outcome of sacral nerve stimulation for fecal incontinence in patients refractory to percutaneous tibial nerve stimulation. Dis Colon Rectum 2013;56:915-920.

42. Tjandra JJ, Chan MK, Yeh CH, et al. Sacral nerve stimulation is more effective than optimal medical therapy for severe fecal incontinence: a randomized, controlled study. Dis Colon Rectum 2008;51:494-502.

43. Wexner SD, Coller JA, Devroede G, et al. Sacral nerve stimulation for fecal incontinence: results of a 120-patient prospective multicenter study. Ann Surg 2010;251:441-449

44. Mellgren A, Wexner SD, Coller JA, et al. Long-term efficacy and safety of sacral nerve stimulation for fecal incontinence. Dis Colon Rectum 2011;54:1065-1075.

45. Altomare DF, Ratto C, Ganio E, et al. Long-term outcome of sacral nerve stimulation for fecal incontinence. Dis Colon Rectum 2009;52:11-17.

46. Ramage L, Qiu S, Kontovounisios C, Tekkis P, et al. A systematic review of sacral nerve stimulation for low anterior resection syndrome. Colorectal Dis 2015;17:762771.

47. Hollingshead JR, Dudding TC, Vaizey CJ. Sacral nerve stimulation for faecal incontinence: results from a single centre over a 10-year period. Colorectal Dis 2011;13:1030-1034.

48. Duelund-Jakobsen J, van Wunnik B, Buntzen S, et al. Functional results and patient satisfaction with sacral nerve stimulation for idiopathic faecal incontinence. Colorectal Dis 2012;14:753-759.

49. Stewart WF, Liberman JN, Sandler RS, et al. Epidemiology of constipation (EPOC) study in the United States: relation of clinical subtypes to sociodemographic features. Am J Gastroenterol 1999;94:3530-3540.

50. Everhart JE, Ruhl CE. Burden of digestive diseases in the United States part II: lower gastrointestinal diseases. Gastroenterology 2009;136:741- 754.

51. Ganio E, Masin A, Ratto C, et al. Short-term sacral nerve stimulation for functional anorectal and urinary disturbances: results in 40 patients: evaluation of a new option for anorectal functional disorders. Dis Colon Rectum 2001;44:1261-1267.

52. Zurbuchen U, Groene J, Otto S, et al. Sacral neuromodulation for fecal incontinence and constipation in adult patients with anorectal malformation- a feasibility study in patients with or without surgical dysgenesis. Int J Colorectal Dis 2014;29:1297-302.

53. Kamm MA, Dudding TC, Melenhorst J, et al. Sacral nerve stimulation for intractable constipation. Neurogastroenterology Gut 2010;59:333-340

54. Gortazar de las Casas S, Rubio-Perez I, Saavedra Ambrosy J, et al. Sacral nerve stimulation for constipation: long-term outcomes. Tech Coloproctol 2019;23:559-564

55. Govaert B, Maeda Y, Alberga J, et al. Medium-term outcome of sacral nerve modulation for constipation. Dis Colon Rectum 2012;55:26-31. 
56. Lu PL, Koppen JN, Orsagh-Yentis DK, et al. Sacral nerve stimulation for constipation and fecal incontinence in children: long-term outcomes, patient benefit, and parent satisfaction. Neurogastroenterology \& motility 2018;30:2e13189

57. Dinning PG, Hunt L, Patton V, et al. Treatment efficacy of sacral nerve stimulation in slow transit constipation: a two-phase, double-blind randomized controlled crossover study. Am J Gastroenterol 2015;110:733- 740.

58. Suttor VP, Prott GM, Hanson RD, et al. Evidence for pelvic floor dyssynergia in patients with irritable bowel syndrome. Dis Colon Rectum 2010;53:156-60

59. Lundby L, Krogh K, Buntzen S, et al. Temporary sacral nerve stimulation for treatment of irritable bowel syndrome: a pilot study. Dis Colon rectum 2008; 51:10748

60. Fassov J, Lunby L, Laurberg S, et al. Three-year follow-up of sacral nerve stimulation for patients with diarrhea- predominant and mixed irritable bowel syndrome. Colorectal Dis 2017; 19:188-193

61. Tipsmark LS, Fassov J, Lundby L et al. Cost effectiveness analysis of sacral nerve stimulation as treatment for severe irritable bowel syndrome. Colorectal Dis 2016;18: 030-036 